\title{
Estrategia didáctica para mejorar la atención en clases en estudiantes de enseñanza secundaria
}

\author{
Didactic strategy to improve attention in classes in secondary school students
}

Estratégia didática para melhorar a atenção nas aulas de alunos do ensino médio

\section{Milagros Lupe Zambrana Mogro}

milagroszmogro@outlook.es

https://orcid.org/0000-0002-3837-9082

Recibido: marzo 2020 Revisado: marzo 2020

Universidad Privada Domingo Savio, Potosí, Bolivia

RESUMEN

En educación la atención en clase se relaciona con el éxito o el fracaso de los estudiantes, ya que este proceso psicológico es esencial para captar y procesar información importante para que ocurra el aprendizaje. El artículo presenta, de forma parcial, una estrategia didáctica para mejorar la atención en clase de los estudiantes de quinto de secundaria. La población estuvo constituida por 25 estudiantes y 7 docentes del "Colegio "Manuel Ascencio Padilla" situado en Potosí, Bolivia. Es una investigación proyectiva con un diseño de campo. Las técnicas para la recolección de los datos fueron la observación no participativa, la entrevista y la encuesta y los instrumentos la guía de observación de la atención en clase, la guía de entrevista a los docentes y el cuestionario para los estudiantes. La estrategia didáctica se diseñó para atender la actualización de los docentes y las necesidades diagnosticadas en los estudiantes en relación a la atención en clase.

Palabras clave:

Atención en clase; enseñanza secundaria; estrategia didáctica; aprendizaje; rendimiento académico
ABSTRACT

In education, attention in class is related to the success or failure of students, since this psychological process is essential to capture and process important information for learning to occur. The article presents, in part, a didactic strategy to improve the attention in class of fifth year high school students. The population consisted of 25 students and 7 teachers from the "Manuel Ascencio Padilla" School located in Potosí, Bolivia. It is a projective investigation with a field design. The techniques for data collection were non-participatory observation, the interview and the survey, and the instruments were the observation guide for attention in class, the teacher interview guide, and the student questionnaire. The didactic strategy was designed to attend to the updating of the teachers and the needs diagnosed in the students in relation to the attention in class.

Key words:

Attention in class; secondary education; didactic strategy; learning; academic performance
Na educação, a atenção nas aulas está relacionada ao sucesso ou fracasso dos alunos, uma vez que esse processo psicológico é essencial para captar e processar informações importantes para que a aprendizagem ocorra. O artigo apresenta, em parte, uma estratégia didática para melhorar o atendimento nas turmas de alunos do quinto ano do ensino médio. A população era composta por 25 alunos e 7 professores da Escola "Manuel Ascencio Padilla" localizada em Potosí, Bolívia. É uma investigação projetiva com desenho de campo. As técnicas de coleta de dados foram a observação não participante, a entrevista e a pesquisa, e os instrumentos foram o guia de observação para atenção em aula, o guia de entrevista do professor e o questionário do aluno. A estratégia didática foi desenhada para atender à atualização dos professores e às necessidades diagnosticadas nos alunos em relação ao atendimento em sala de aula.

Palavras-chave:

Atenção nas aulas; Educação secundária; estratégia didática; Aprendendo; rendimento acadêmico 


\section{INTRODUCCIÓN}

La atención es un mecanismo cerebral que permite procesar los estímulos, pensamientos o acciones relevantes e ignorar los irrelevantes o distractores (Gazzaniga, Ivry y Mangun, 2013). Es necesario que exista la atención porque el entorno cambia constantemente y la capacidad del cerebro tiene límites para procesar la información y llevar a cabo de forma eficaz más de una tarea cognitiva a la vez. Los mecanismos neurológicos que permiten seleccionar los estímulos más relevantes es lo que se conoce como atención.

En el campo educativo se entiende la atención como un proceso psicológico básico y se considera como un elemento esencial para captar y procesar información (Van de Heijden, 1992; Laberge, 1995). Los estudiantes que tienen una baja atención en clases presentan dificultades para el procesamiento de la información oral o escrita y esto influye en su rendimiento académico.

La atención está ligada al éxito o fracaso escolar; estudiantes con las mejores calificaciones exhiben una mejor atención; en cambio los estudiantes distraídos e inquietos durante la clase presentan bajo rendimiento, la atención repercute en la adquisición de competencias escolares (Boujon y Quaireau, 1999 y Barca, 2005).

Por consiguiente la atención es muy importante en el proceso de aprendizaje porque es un prerrequisito para que ocurran los procesos de consolidación, mantenimiento y recuperación de la información. Las investigaciones de Syka y Merzenich, (2005), han demostrado que la atención es básica para la creación de nuevas conexiones neuronales y para la formación de circuitos cerebrales estables, que conforman las estructuras de los nuevos aprendizajes.

Al respecto Lupón, Torrents y Quevedo (2012) agregan que la atención permite orientar y seleccionar de forma precisa los aspectos del entorno que son relevantes y que requieren elaboración cognitiva, separándolos de los que no son irrelevantes, que por el contrario reciben un procesamiento mínimo o nulo. Así se asume una capacidad limitada puesto que ante la imposibilidad de atender a todos los estímulos la atención es el proceso adaptativo que selecciona lo más importante para percibirlos mejor y evitar sobrecargas.

La atención en el aula de clase permite dar relevancia a los aspectos educativos y desestimar los elementos distractores que no están relacionados con la actividad de enseñanza y aprendizaje.

Los tipos de atención que se pueden encontrar en el acto educativo se clasifican en:

1. Atención como filtro: se refiere a no sobrecargar la información que se adquiere, es necesario obviar la información irrelevante, que solo obstaculiza el poder procesar información de mucha importancia que sirve en un determinado momento y para una determinada actividad (Hernández, 2012).

2. Atención selectiva: solo se concientiza la información importante y necesaria, de todos los estímulos que capta el cerebro solo se seleccionan los que interesan para un objetivo en concreto (Pérez, 2008).

3. Atención sostenida: permite mantener un estado de vigilancia, focalizada en un estímulo específico durante períodos prolongados (Vaello Orts, 2011)

4. Atención dividida: se refiere a la capacidad de ejecutar con éxito más de una tarea a la vez, prestando atención a dos o más canales de información.

5. Atención involuntaria: es cuando se obtiene la información automáticamente, sucede cuando un estímulo captura la atención (Hernández, 2012). 
6. Atención voluntaria: es la que permite concentrarse de manera intencional como producto de la voluntad y la decisión del individuo ((Hernández, 2012)

Los diferentes tipos de atención están presentes en diferentes momentos del proceso de enseñanza aprendizaje, por lo tanto es conveniente identificarlas para suprimir cualquier efecto negativo que afecte la captación y retención información importante o relevante.

Hernán Gascón y Paredes (2008), Navas (2010) y Osorio (2012) coinciden en mencionar que los elementos que se deben atender para construir una propuesta que permita desarrollar una atención efectiva en el aula son: planificación del tiempo, uso de métodos, técnicas y estrategias de enseñanza activa, normas de convivencia consensuadas, uso de la tecnología, desarrollo de valores como el respeto y la confianza, comunicación y participación efectiva entre docentes y alumnos.

Todos estos elementos deben ser contemplados para construir una propuesta que busque mejorar la atención en clase y por consiguiente optimizar el proceso de enseñanza aprendizaje y rendimiento académico de la población estudiantil.

Como producto de las prácticas profesionales en el colegio "Manuel Ascencio Padilla" de la ciudad de Potosí, se logró observar la conducta de los estudiantes del quinto de educación secundaria y sus problemas de atención en clases. Los estudiantes constantemente se distraen con ruidos que ocurren fuera del aula, hacen uso continuo de los celulares dentro del aula en clases para revisar su Facebook o WhatsApp, juegan con los celulares, no responden a las preguntas que realiza el docente, no toman notas durante la clase.

Además de las observaciones anteriores se conversó con algunos estudiantes y ellos hicieron referencia que no prestan atención a la clase porque trabajan toda la noche en la mina y esto les ocasiona cansancio y falta de interés; como consecuencia tienen muy bajo rendimiento escolar.

A partir de toda la información recabada en la experiencia práctica surgió el interés de diagnosticar el estado de la atención en clases y con base al diagnóstico buscar soluciones para atender esta situación, por consiguiente se formula el siguiente problema:

¿Cuáles son las características de una estrategia didáctica que permita mejorar la atención en clase de los estudiantes de quinto de secundaria del Colegio "Manuel Ascencio Padilla" de la ciudad de Potosí en Bolivia?

Objetivo de investigación: Proponer una estrategia didáctica para el mejoramiento de la atención en clase de los estudiantes de quinto de secundaria del Colegio "Manuel Ascencio Padilla" de la ciudad de Potosí en Bolivia.

\section{MÉTODO}

Es una investigación proyectiva en la cual se diseña la propuesta de una estrategia didáctica fundamentada en el diagnóstico. El enfoque es mixto porque se obtienen y procesan datos de forma cualitativa y cuantitativa, el diseño es de campo, pues los datos se recolectaron en el contexto natural.

El contexto en el cual se desarrolla la investigación es el colegio "Manuel Ascencio Padilla" ubicado en la ciudad de Potosí en Bolivia. La población la conforman 25 estudiantes entre varones y mujeres que cursan el quinto de secundaria y 7 profesores que se desempeñan en este curso.

Las técnicas para la recolección de los datos fueron la observación no participativa, la entrevista y la encuesta. Los instrumentos estuvieron constituidos por: (a) la guía de observación 
compuesta por 10 ítems que capta la atención en clases, en sus tres dimensiones la participación oral, las actividades escritas y el control de distractores. Este instrumento se utilizó durante las clases de Química. Filosofía, Matemáticas, Literatura, Física, Psicología, Historia, Religión, Cívica y Biología. (b) Una guía de entrevista semi estructurada, con 17 ítems, para conocer la percepción de los docentes acerca de la atención en clase que presentan los estudiantes. (c) El cuestionario estructurado con 9 ítems, se aplicó a los estudiantes para medir la atención en clases de sus compañeros desde su percepción.

La variable dependiente de la investigación es la atención en clases y su operacionalización con tres dimensiones se observa en el Cuadro 1.

Cuadro 1. Operacionalización de la variable dependiente.

\begin{tabular}{|c|c|c|c|c|c|}
\hline \multirow[b]{2}{*}{$\begin{array}{c}\text { Variable } \\
\text { Definición operativa }\end{array}$} & \multirow[b]{2}{*}{ Dimensiones } & \multirow[b]{2}{*}{ Indicadores } & \multicolumn{3}{|c|}{ Instrumentos } \\
\hline & & & 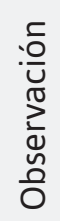 & 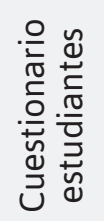 & 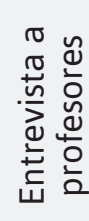 \\
\hline \multirow{10}{*}{$\begin{array}{l}\text { La atención en el aula } \\
\text { Es la capacidad de seleccionar } \\
\text { la información de forma precisa } \\
\text { de los temas y actividades } \\
\text { efectuadas en el ambiente o } \\
\text { entorno en el cual se desarrolla el } \\
\text { proceso enseñanza - aprendizaje, } \\
\text { evidenciándose a partir de la } \\
\text { participación en las actividades } \\
\text { y la disposición del estudiante, } \\
\text { cuyo efecto se evidencia en el } \\
\text { rendimiento académico. }\end{array}$} & \multirow[t]{6}{*}{$\begin{array}{l}\text { Participación de } \\
\text { forma oral }\end{array}$} & $\begin{array}{l}\text { Participa de los temas que se } \\
\text { desarrollan en el aula. }\end{array}$ & $x$ & $x$ & $x$ \\
\hline & & $\begin{array}{l}\text { Realiza preguntas } \\
\text { relacionadas con el tema. }\end{array}$ & $x$ & $x$ & $x$ \\
\hline & & $\begin{array}{l}\text { Propone actividades } \\
\text { relacionadas con el tema. }\end{array}$ & $x$ & $x$ & $x$ \\
\hline & & $\begin{array}{l}\text { Responde a las preguntas de } \\
\text { manera pertinente de acuerdo } \\
\text { al tema. }\end{array}$ & $x$ & $x$ & $x$ \\
\hline & & $\begin{array}{l}\text { Emite opiniones referidas al } \\
\text { tema que se trata }\end{array}$ & $x$ & $x$ & $x$ \\
\hline & & $\begin{array}{l}\text { Realiza un resumen verbal del } \\
\text { tema al final de la clase. }\end{array}$ & $x$ & $x$ & $x$ \\
\hline & \multirow[t]{2}{*}{ Actividades escritas } & $\begin{array}{l}\text { Toma apuntes del tema que } \\
\text { se trata en el aula. }\end{array}$ & $x$ & $x$ & $x$ \\
\hline & & $\begin{array}{l}\text { Realiza trabajos escritos } \\
\text { propuestos por el profesor en } \\
\text { el aula. }\end{array}$ & $x$ & $x$ & $x$ \\
\hline & \multirow{2}{*}{$\begin{array}{l}\text { Aislamiento } \\
\text { de elementos } \\
\text { distractores }\end{array}$} & Cumple las reglas en el aula & $x$ & $x$ & $x$ \\
\hline & & $\begin{array}{l}\text { Asume postura física corporal } \\
\text { dirigida al maestro. }\end{array}$ & $x$ & $x$ & $x$ \\
\hline
\end{tabular}




\section{RESULTADOS}

Los resultados se presentarán de acuerdo a los instrumentos aplicados.

\section{Guía de observación}

Este instrumento se administró para observar la conducta en el aula, con respecto a la atención en clases, de los estudiantes del quinto curso de secundaria del colegio "Manuel Ascencio Padilla", la observación se realizó durante el desarrollo de 10 clases en materias diferentes. (Ver Gráfico 1).

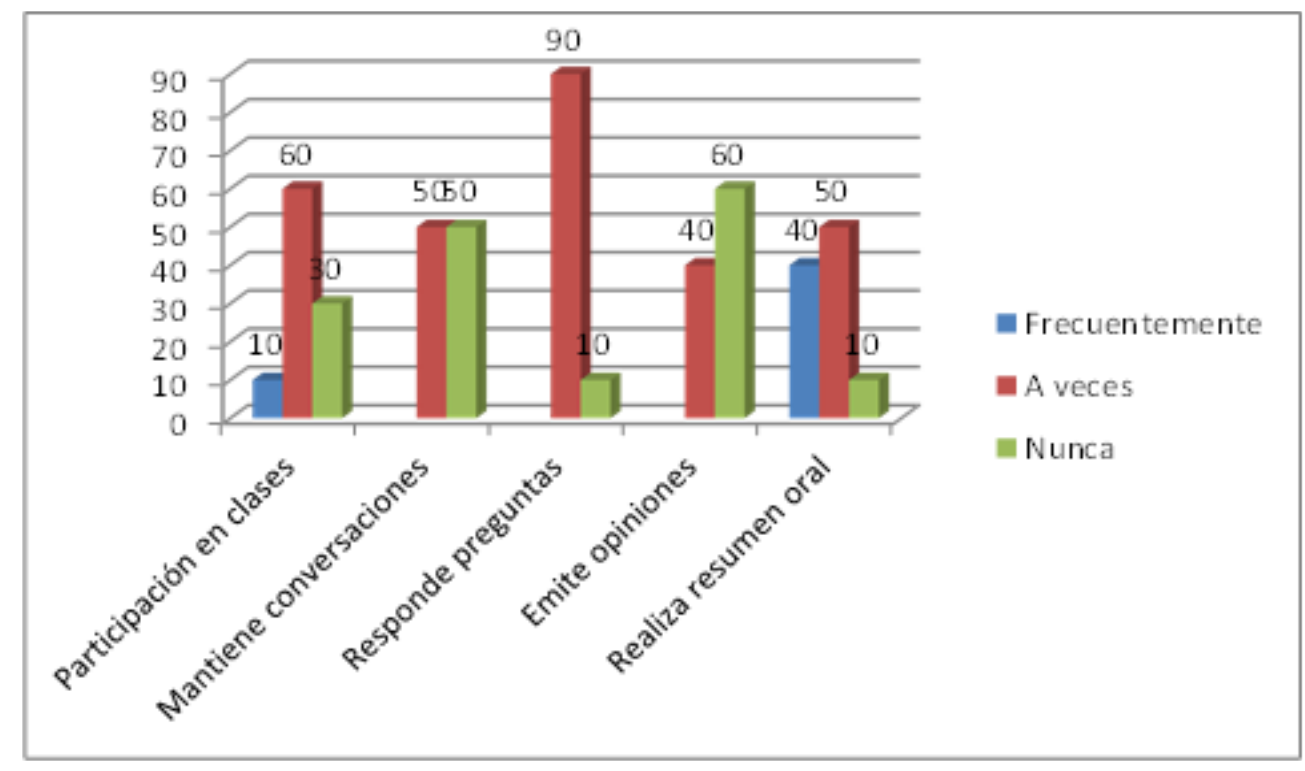

Gráfico 1. Dimensión: Participación de forma oral.

En la dimensión Participación de forma oral, los indicadores con mayor debilidad son el de emitir opiniones y mantener conversaciones de la temática desarrollada en la clase. El 60\% nunca emite opiniones y el $40 \%$ opina a veces; el $50 \%$ nunca mantiene conversaciones sobre los temas de la clase el otro $50 \%$ a veces; el $30 \%$ participa $60 \%$ lo hace a veces y solo un $10 \%$ tiene participación activa en la clase. Cuando se les solicita el resumen oral de la clase el $40 \%$ lo hace frecuentemente, el $50 \%$ a veces y el $10 \%$ nunca lo hace.
Estos resultados permiten captar que los estudiantes de quinto de secundaria presentan dificultades y se observa una baja participación en la clase, de acuerdo a lo revisado en el marco teórico, algunos parámetros que permiten conocer si los estudiantes prestan o no atención en clases están referidos a la participación.

La dimensión actividades escritas capta la atención que prestan los estudiantes para tomar notas de las explicaciones y la realización de trabajos escritos, los resultados en el Gráfico 2. 


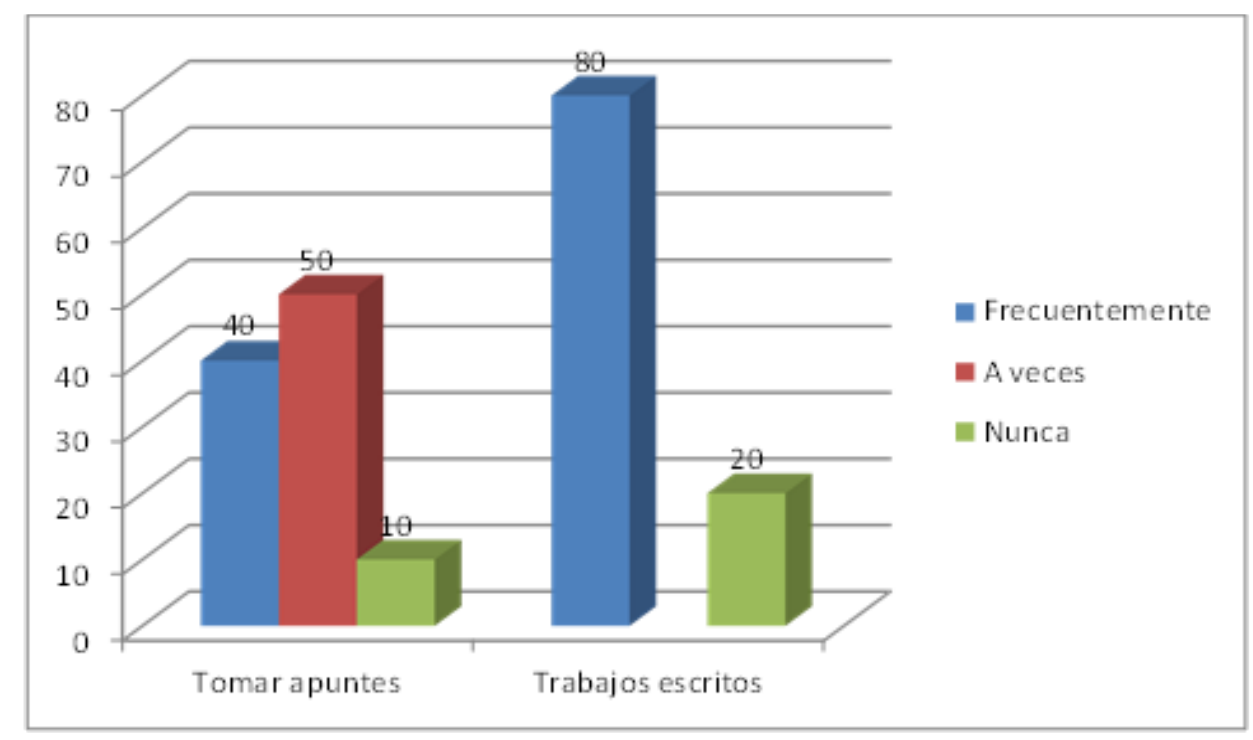

Gráfico 2. Actividades escritas.

La dimensión actividades escritas tiene dos indicadores el de tomar apuntes y la realización de trabajos. El 50\% de los estudiantes a veces toman apuntes en clases, aunque utilizan los celulares para guardar la información de la pizarra o de las láminas, pero esto no asegura la misma atención que ir tomando apuntes escritos a medida que el profesor desarrolla el tema. Los docentes permiten el uso de los celulares en clase y este es otro motivo de distracción. El $40 \%$ si toman apuntes de forma frecuente y el $10 \%$ nunca toman apuntes.

El $80 \%$ realiza trabajos escritos cuando el profesor lo solicita el $20 \%$ nunca realiza trabajos escritos que el profesor asigna dentro del aula.

Tanto en la dimensión de participación oral como en la ejecución de trabajos escritos, cuando el docente solicita al estudiante el resumen oral o la realización de algún ejercicio por escrito, la respuesta de los estudiantes es positiva y de forma regular ejecutan lo solicitado, lo realizan por la presión que hace el profesor a su actuación.

Esto les perjudica bastante en cuanto a su rendimiento académico diario en las clases, por estar hablando o por el simpe hecho de grabar en su celular lo que el profesor explica y no toman apuntes, el no tomar apuntes y hablar entre ellos y no cumplir con las tareas refleja que ellos no prestan atención y al final de gestión el rendimiento de los estudiantes es poco favorable.

La dimensión aislamiento de elementos distractores se construye con tres indicadores: la puntualidad para incorporarse a la clase, el interrumpir la clase hablando acerca de otras temáticas diferentes al tema de la clase y el control consciente de los distractores al inicio de la clase, los resultados se muestran en el Gráfico 3. 


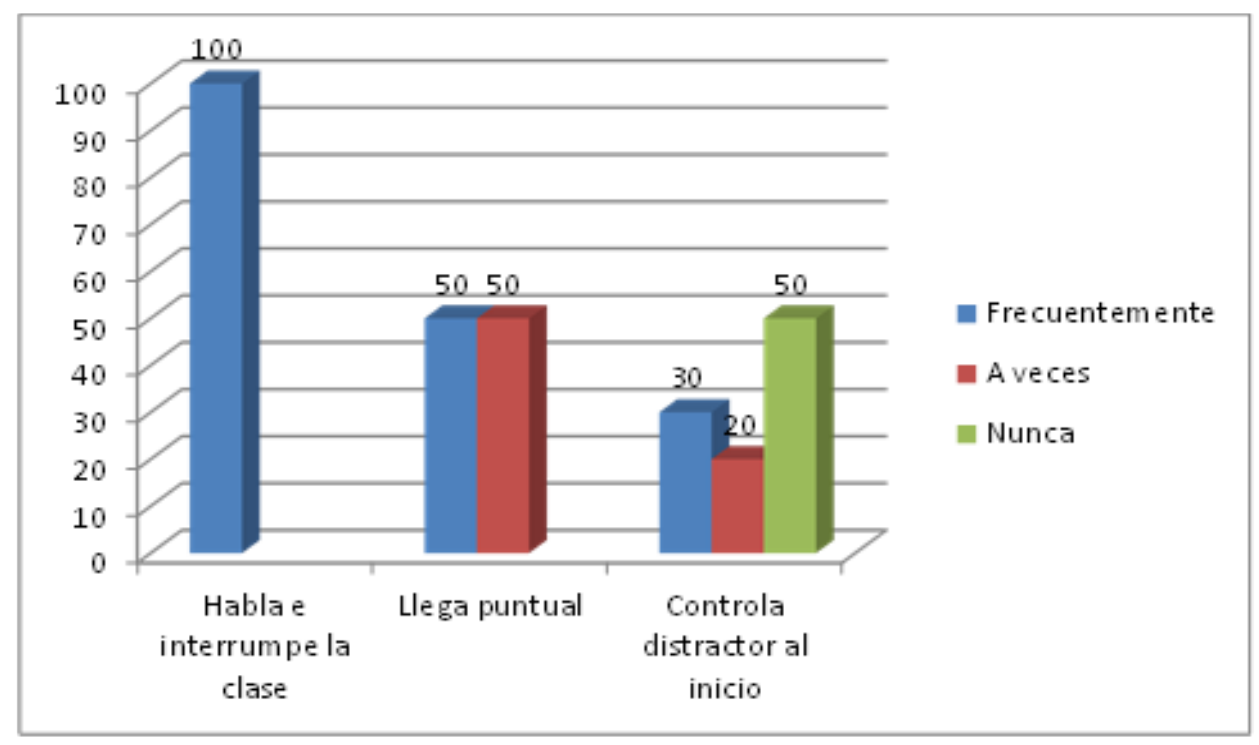

Gráfico 3. Dimensión aislamiento de elementos distractores.

La totalidad de los estudiantes en un momento u otro se distraen y comienzan conversaciones de otros aspectos que no son los desarrollados en la clase. El $50 \%$ de los estudiantes se incorpora de forma puntual al aula de clases el otro $50 \%$ lo hace a veces, esto es un distractor porque cada vez que entra un estudiante todos están mirando hacia la puerta. Con respecto al control de elementos distractores $50 \%$ nunca lo hacen el $30 \%$ lo hace frecuentemente y el $20 \%$ lo logra a veces.

\section{Guía de entrevista}

La guía de entrevista se aplicó a los docentes que trabajan con los estudiantes de quinto de secundaria del colegio "Manuel Ascencio Padilla" de la ciudad de Potosí; está constituida por las mismas dimensiones que la guía de observación, el objetivo de este instrumento es captar desde la percepción del docente las dimensiones de la atención.

Los docentes están de acuerdo que la atención en clases es importante, la respuesta de la mayoría de los profesores fue que es primordial para que los estudiantes puedan tener un mejor aprendizaje y un mejor rendimiento académico al finalizar la gestión. Se les preguntó acerca de las actividades que ellos realizan para captar mejor la atención de los estudiantes en su mayoría la respuesta fue que les hacen charlas, los profesores mencionan que por el tiempo de las clases no es factible hacer actividades recreativas.

Al considerar la participación de los estudiantes en clases, los docentes están de acuerdo en declarar que los estudiantes participan de forma obligada y no por voluntad propia ellos los incentivan $u$ obligan a participar de las clases diciéndoles que su participación será calificada, en otros casos los estudiantes no suelen participar. Así mismo los estudiantes no suelen emitir opiniones, ni realizan preguntas de sus dudas y tampoco responden a preguntas hechas por los profesores por miedo a equivocarse o porque no ponen atención y no saben que decir, en una que otra materia en un mínimo porcentaje los alumnos preguntas esto por tratarse de materias relacionadas con el cálculo.

En lo que respecta a la segunda dimensión los docentes coinciden que solo una minoría de los estudiantes toma apuntes en las materias teóricas, y en mayor cantidad en las materias prácticas. Los estudiantes usan el celular para grabar y fotografiar las explicaciones o asignaciones para realizar en la casa. Con respecto al trabajo en el 
aula si consideran que los estudiantes realizan las asignaciones de trabajos escritos. Consideran que los estudiantes se motivan solo si las actividades tienen impacto en su calificación.

Para finalizar con los resultados de la entrevista a los profesores, consideran que las reglas que menos cumplen sus estudiantes, son el llegar puntal al aula de clases el uso de los celulares fue de que llegan tarde a clases, el uso constante de los celulares, el incumplimiento de la entrega de cuadernos, trabajos o asignaciones. El incumplimiento de estas asignaciones baja las calificaciones.

\section{Cuestionario para los estudiantes}

Con este instrumento mide la atención en clases de los estudiantes desde la percepción de sus compañeros de clases.

Los resultados para la primera dimensión, participación de forma oral, en el Gráfico 4.

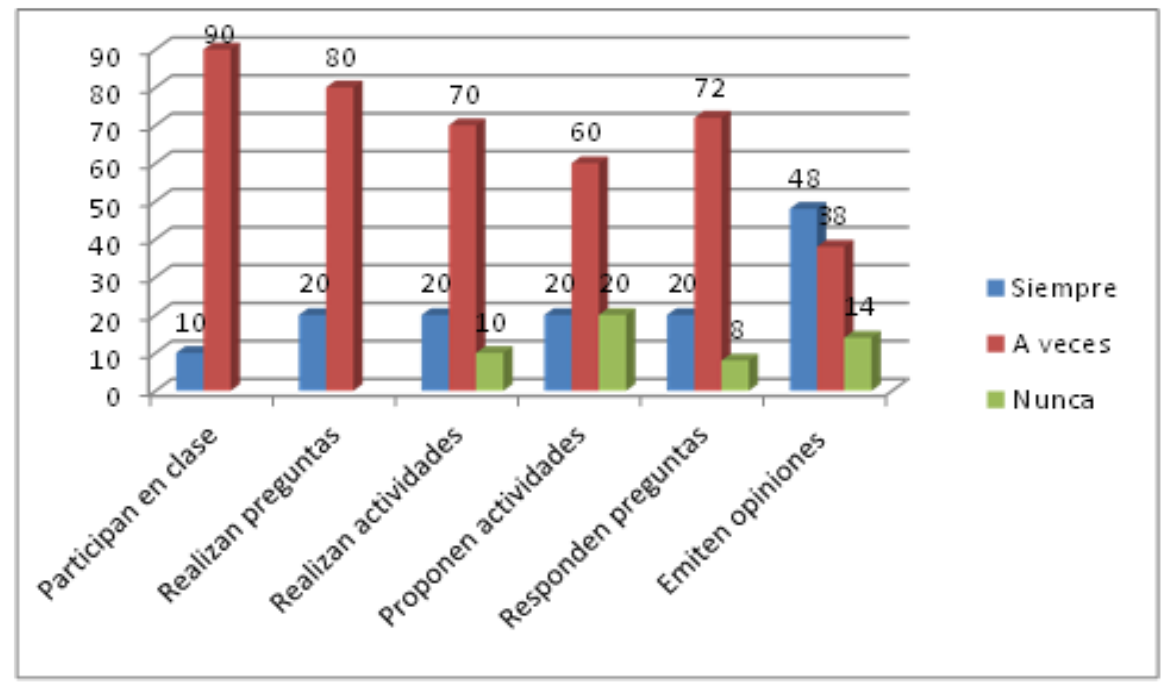

Gráfico 4. Dimensión: Participación de forma oral desde la percepción de los estudiantes.

La participación de los estudiantes en clases el $90 \%$ lo hacen a veces, pero siempre que el docente se los requiere, solo el $10 \%$ participan de forma espontánea. Las respuestas de los estudiantes se ubican en "A veces", solo la de emitir opinión de forma libre $48 \%$ consideran que tienen libertad de emitir opiniones, $38 \%$ a veces pueden hacerlo y el $14 \%$ que nunca tienen libertad de emitir su opinión.

La segunda dimensión acerca de la realización de actividades escritas, se resume en el Gráfico 5. 


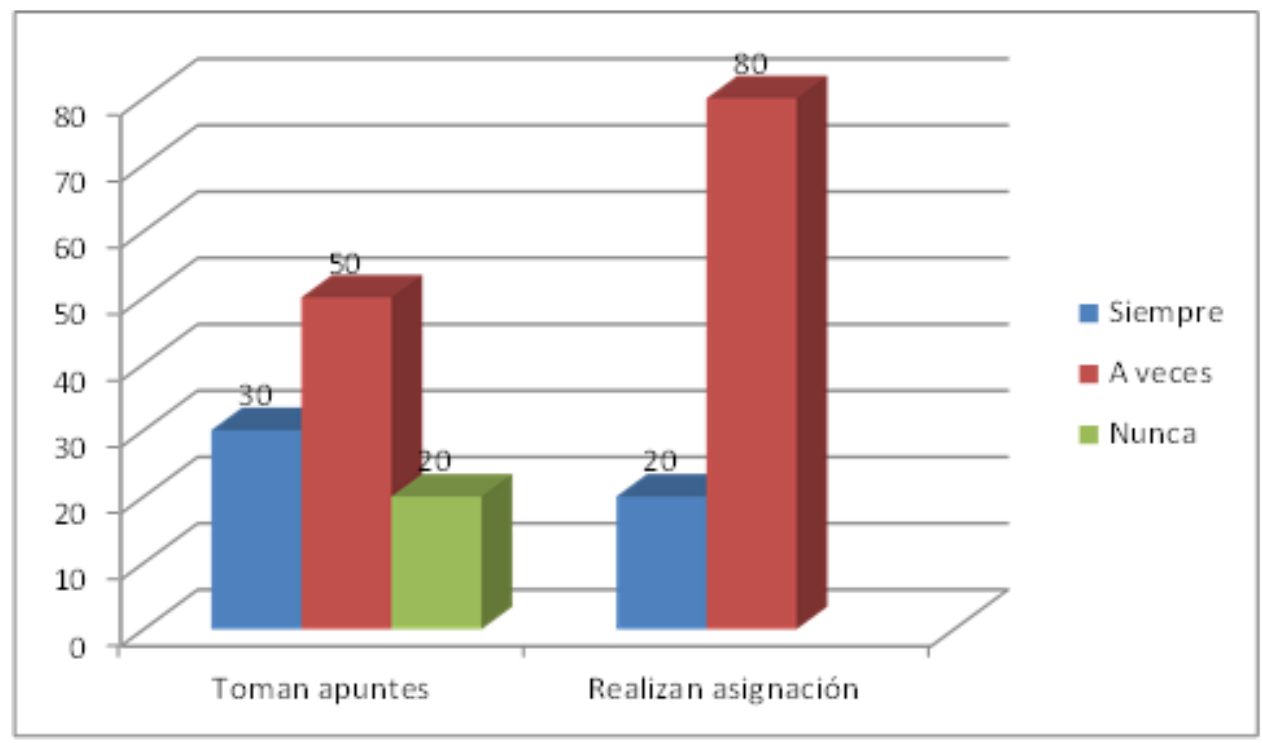

Gráfico 5. Actividades escritas desde la percepción de los estudiantes.

El $50 \%$ de los estudiantes consideran que sus compañeros a veces toman apuntes, el 30\% considera que siempre toman apuntes, el $20 \%$ consideran que nunca toman apuntes.

$\mathrm{Si}$ los compañeros de clase realizan asignaciones o tareas escritas en el aula, el $80 \%$ de los estudiantes consideran que las realizan a veces y el $20 \%$ que siempre las realizan.

El $52 \%$ de los estudiantes consideran que los docentes tienen reglas para la convivencia en clases, $48 \%$ consideran que no hay reglas en el aula de clases y por esa razón hablan entre ellos, usan sus teléfonos celulares y pueden ser impuntuales para llegar a la clase.

Con base en los resultados se diseña la siguiente estrategia didáctica para mejorar la atención en clases de los estudiantes de quinto de secundaria del colegio "Manuel Ascencio Padilla" ubicado en la ciudad de Potosí.

\section{Propuesta "Atendemos y Entendemos"}

\section{Justificación}

La estrategia didáctica son los procedimientos, técnicas, métodos, actividades y materiales que se organizan para desarrollar el proceso de enseñanza y aprendizaje adaptándose a las características y necesidades de los participantes, constituyendo las practicas pedagógicas que tienen por objetivo una eficaz acción pedagógica del docente (Ignacio, 2014 y Choque, 2016).

La propuesta se fundamenta en principios de la didáctica, la psicología y la pedagogía.

Según Herrán Gascón, de la y Paredes (2008), desde la didáctica se atiende los principios de:

1. Individualidad al considerar a cada estudiante como un ser único con características, intereses y aptitudes propias.

2. Socialización: la educación es un hecho social es por eso que la se debe centrar y socializar en el educando dándole la oportunidad a que se integre a la comunidad.

3. Actividad: asegura la participación y la atención, se consideran los procedimientos de enseñanza activa. En esta propuesta se diseñan actividades recreativas que aseguran la actividad.

4. La creatividad para entender las situaciones desde diferentes perspectivas. 
Según Osorio (2012) desde la pedagogía se atiende los principios de:

1. Trabajo colaborativo para el logro de objetivos de la organización, de los docentes y estudiantes.

2. Centrar todas centrar todos los procesos de aprendizaje en las características e intereses de los estudiantes.

3. Utilizar la evaluación como una posibilidad de aprendizaje.

Desde lo psicológico la propuesta atiende el desarrollo de un ambiente educativo en el cual se genere la confianza, el respeto y la valoración de los estudiantes y docentes para generar un estado emocional de bienestar.
La propuesta consta de talleres para actualizar a los docentes acerca de las estrategias más efectivas para lograr la atención en clases de los estudiantes y sesiones de trabajo planificadas para los estudiantes para la adquisición de herramientas cognitivas que le permitan controlar su entorno y aumentar su atención en clases.

\section{Objetivos}

Actualizar a los docentes en estrategias didácticas para mejorar la atención en clases.

Atender las necesidades de los estudiantes para el logro de una atención activa y efectiva.

A continuación se presenta de forma parcial los talleres para el personal docente y para los estudiantes de quinto de secundaria.

\section{Talleres para docentes}

\section{Taller 1. La Atención}

Objetivo: Fortalecer los conocimientos de la atención a través de actividades participativas para apoyarlos en su proceso de enseñanza aprendizaje.

Contenido: La atención, definiciones, características, factores psicológicos y del ambiente, rol del docente, estrategias creativas para la atención.

Tiempo: $50^{\prime}$

Participantes: 7 docentes

Materiales y recursos: Marcadores, papelografos, computadora, presentaciones.

\section{Actividad de Inicio}

Cadena de nombres: todos los participantes incluido el facilitador, uno de los profesores comienza diciendo el nombre de la persona que tiene a su derecha, menciona vine con... y así sucesivamente lo hacen los demás hasta culminar en el facilitador, el objetivo es conocer a los participantes para llamarlos por su nombre en todo el taller y poder entrar en confianza.

\section{Actividad de Desarrollo}

El buscador: Los participantes estén sentados alejados, uno de los participantes que hace el papel del buscador, este debe buscar de manera silenciosa y disimulada a los participantes que estén distraídos hablando y sin poner atención una vez que los identifique el buscador les pide uno por uno que pasen al frente y le realiza diferentes preguntas de lo que anteriormente se estaba hablando, esto con el fin de comprobar el grado de atención, de la misma manera esta actividad servirá para explicar los diferentes tipos de atención en general, definición y sus características. Realizar preguntas acerca del contenido.

Actividad de cierre: Se realiza la evaluación para ello cada participante elabora una pregunta y otro participante la responde. El facilitador tiene tarjetas impresas. 


\section{Taller 2. Mejorando la Atención}

Objetivo: Actualizar los conocimientos en estrategias para mejorar la atención en clases.

Contenido: Estrategias para desarrollar la atención, factores que intervienen en la atención en clases, bases psicológicas, pedagógicas y didácticas de la atención en clases.

Tiempo: $35^{\prime}$

Participantes: 7 docentes

Materiales y recursos: Marcadores, papelografos, computadora, presentaciones.

\section{Actividad de Inicio}

El curioso: los participantes incluyendo el facilitador se colocan en círculo, el facilitador pregunta a cada uno acerca de las actividades realizadas los días después del primer taller, se concluye con un resumen de todas las respuestas.

\section{Actividad de Desarrollo}

Trabajaran en grupo, reciben del facilitador una hoja con los elementos que permiten mejorar la atención en clases, tienen 5 minutos para conocer cada elemento, deben diseñar una estrategia para que cada elemento sea haga presente en el salón de clases. Los elementos son: 1. Eliminación de fuentes de distracción, los profesores antes de iniciar su clase deberán alejar de los estudiantes elementos distractores, en el taller los profesores trabajaran haciendo un simulacro como si ya estuvieran en clases. 2. Gestionar el tiempo, se debe planificar cada momento de la clase. 3. Realizar juegos de memoria, lógica y atención; los juegos pueden tener el contenido o temática que se está desarrollando. 4. Realizar organizadores de contenido de la temática desarrollada. Pueden utilizar esquemas, mapas mentales, mapas conceptuales, resúmenes orales, resúmenes escritos, fichas con diferentes colores, en este taller debe hacerse con el contenido de la Atención. 5.- Utilizar recursos y materiales que ya formen parte del estudiante, puede ser programar un video o una exposición fotográfica, la cartelera, elaborar un blog, elaborar un podcast.

Actividad de cierre: Se realiza la evaluación de la dinámica se exponen los productos del taller.

\section{Taller 3. La tecnología en el aula}

Objetivo: Actualizar los conocimientos en el papel de la tecnología como herramienta educativa.

Contenido: Tecnologías de la Información y la Comunicación (TIC), Tecnología de Aprendizaje y la Comunicación (TAC), Tecnología del Empoderamiento y la participación (TEP). Motores de búsqueda, repositorios. Videos educativos. Construcción de productos educativos a través de la tecnología: blogs, wiki, podcast, Instagram, twitter.

Tiempo: 60'

Participantes: 7 docentes

Materiales y recursos: Marcadores, papelografos, computadora, presentaciones, teléfonos celulares inteligentes, conexión a internet.

\section{Actividad de Inicio}

Marea sube marea baja" de 3 a 5 minutos.

\section{Actividad de Desarrollo}

Se realizan preguntas acerca del contenido del taller. Los participantes deben planificar una actividad educativa con el uso del celular en la cual los estudiantes deban buscar, evaluar, seleccionar, procesar y difundir una información de acuerdo a un tema (en este taller el contenido puede ser la atención o el uso de las tecnologías).

Actividad de cierre: Se realiza la evaluación de la dinámica se resumen los logros del taller. 


\section{Taller 4. Las reglas de oro}

Objetivo: Diseñar estrategias para la negociación de normas de convivencia

Contenido: Normas de convivencia, autoestima, clima del aula, valores: respeto, confianza. Factores que influyen en la creación y cumplimiento de normas.

Tiempo: $60^{\prime}$

Participantes: 7 docentes

Materiales y recursos: Marcadores, papelografos, computadora, presentaciones.

\section{Actividad de Inicio}

"Las estatuas"; el facilitador pedirá a todos los participantes que se pongan de pie y que vayan caminando por todo el curso moviendo los brazos, los pies y relajando la cabeza y el cuello, después de un corto tiempo el facilitador les pide que cuando el mencione una palabra todos deberán formar estatuas que describan la palabra mencionada por el facilitador, se debe repetir varias veces el mismo ejercicio con diferentes palabras.

\section{Actividad de Desarrollo}

Se realizan preguntas acerca del contenido del taller. Los participantes deben planificar una simulación para generar y negociar normas de convivencia en el aula. Se destaca la importancia de los valores que deben estar presentes en la comunicación y participación en el aula. Se construirán materiales el docente o docentes junto a sus estudiantes que permitan el seguimiento de las normas de convivencia.

Por ejemplo para la puntualidad realizarán 5 carteles de colores diferentes para cada día, en el cartel estará escrito un consejo diferente aparte de decir que ya no pueden pasar, esto servirá a los estudiantes a llegar puntuales a clases y a no interrumpir llegando tarde. Se sugiere asignar un lugar para colocar los celulares o un porta celular.

Actividad de cierre: Se realiza la evaluación de la dinámica se resumen los logros del taller se presentan las normas de diferentes formas con dibujos o frases cortas.

\section{Sesiones de trabajo para los estudiantes}

\section{Sesión 1. Atención en clases}

Objetivo: Reflexionar acerca de la importancia de la atención en las clases

Contenido: Atención en clases, definición características, factores que influyen, ventajas, clima del aula.

Tiempo: $60^{\prime}$

Participantes: 25 estudiantes de quinto de secundaria.

Materiales y recursos: Marcadores, papelógrafos, computadora, presentaciones, sopa de letras.

\section{Actividad de Inicio}

El facilitador inicia con una dinámica de grupo que genere la atención, por ejemplo la dinámica del rumor.

\section{Actividad de Desarrollo}

Se realizan preguntas acerca del contenido del taller. Los participantes deben planificar una simulación de un caso de atención en clases y otro que no.

Proporcionar a un estudiante un papelografo con una sopa de letras y pedirle que la resuelva en un tiempo determinado; asimismo otros estudiantes trataran de distraerlo para que no logre la solución en el tiempo previsto. Reflexionar cuales son las conductas que reconocen como propias, explicación de las causas y consecuencias o efectos. Cuáles conductas están dispuestos a cambiar. Resumir los resultados en un organizador de información.

Actividad de cierre: Se la evaluación de la dinámica se resumen los logros de la sesión de trabajo se presentan las conclusiones o acuerdos. 


\section{Sesión 2. ¿Cómo participo?}

Objetivo: Reflexionar acerca de la importancia de la atención en las clases.

Contenido: Participación en clase, ventajas de la participación, normas para la participación, consecuencias de la no participación en clases.

Tiempo: $60^{\prime}$

Participantes: 25 estudiantes de quinto de secundaria.

Materiales y recursos: Marcadores, papel, computadora, presentaciones.

\section{Actividad de Inicio}

El facilitador inicia con una dinámica de grupo que genere la participación, por ejemplo la dinámica de los animales.

\section{Actividad de Desarrollo}

Se realizan preguntas acerca del contenido del taller. Los participantes deben planificar una simulación de un caso de participación en clases y otro que no.

Reflexionar cuales son las conductas que reconocen como propias, explicación de las causas y consecuencias o efectos. Cuáles conductas están dispuestos a cambiar. Resumir los resultados en un organizador de información.

Actividad de cierre: Se realiza la evaluación de la dinámica se resumen los logros de la sesión de trabajo se presentan las conclusiones o acuerdos.

\section{Sesión 3. Apuntes}

Objetivo: Reflexionar acerca de la importancia de tomar apuntes durante la clase.

Contenido: Apuntes o notas de clase, tipos: organizadores, esquemas, mapas mentales, mapas conceptuales, ventajas de los apuntes, consecuencias de no tomar apuntes durante la clase. Uso de los colores en los apuntes.

Tiempo: $60^{\prime}$

Participantes: 25 estudiantes de quinto de secundaria.

Materiales y recursos: Marcadores, papel, computadora, presentaciones, cuadernillos, lápices o bolígrafos para cada estudiante.

\section{Actividad de Inicio}

El facilitador inicia con una dinámica de grupo que genere la participación, por ejemplo la dinámica de la risa y la seriedad, de acuerdo a una palabra del facilitador.

\section{Actividad de Desarrollo}

Se hace una dinámica de dictado rápido se pide a los estudiantes que copien en sus cuadernillos de la manera más exacta posible, luego se lee y se discrimina cual tuvo el mejor dictado. Se discute por qué lo logró ¿si uso abreviaciones?

Se realizan preguntas acerca del contenido del taller. Se dividen en grupo y a cada grupo se le pide que discuta.

Actividad de cierre: Se realiza la evaluación de la dinámica se resumen los logros de la sesión de trabajo se presentan las conclusiones o acuerdos. 


\section{Sesión 4. Distractores}

Objetivo: Reflexionar acerca de los distractores que impiden la atención en las clases.

Contenido: Distractores en el aula, identificación - concientización - control o regulación del distractor. Desventajas de la distracción en las clases. Tipos de distractores: uso del celular

Tiempo: $60^{\prime}$

Participantes: 25 estudiantes de quinto de secundaria.

Materiales y recursos: Marcadores, papel, computadora, presentaciones

Actividad de Inicio

El facilitador inicia con una dinámica de grupo que genere trabajo en equipo por ejemplo: la cuerda.

\section{Actividad de Desarrollo}

Se realizan preguntas acerca del contenido del taller. Los participantes deben planificar una simulación de distractores en clases y sus consecuencias.

Reflexionar cuales son las conductas que reconocen como propias, explicación de las causas y consecuencias o efectos. Cuáles conductas están dispuestos a cambiar. Resumir los resultados en un organizador de información.

Actividad de cierre: Se realiza la evaluación de la dinámica se resumen los logros de la sesión de trabajo se presentan las conclusiones o acuerdos.

\section{Sesión 5. Somos un equipo}

Objetivo: Reflexionar acerca de la importancia del trabajo colaborativo o trabajo en equipo para conseguir mejores resultados. Fortalecer las técnicas del trabajo en equipo.

Contenido: El trabajo en equipo, tipos de equipo, normas para el trabajo en equipo, ventajas del trabajo en equipo, conflictos y forma de solucionarlos, toma de decisiones en el equipo.

Tiempo: $60^{\prime}$

Participantes: 25 estudiantes de quinto de secundaria.

Materiales y recursos: Marcadores, papel, computadora, presentaciones, lápices de colores, hojas blancas.

\section{Actividad de Inicio}

El facilitador inicia con una dinámica La Mentira. El facilitador pide que todos los participantes se pongan de pie y formen un circulo, el facilitador debe empezar actuando algo de su preferencia, la persona que está a su derecha le dice por su nombre y le pregunta " ¿qué estás haciendo?" esta persona responde que está haciendo algo completamente diferente, por ejemplo el facilitador simula que está comiendo una manzana y dice al que le pregunto " estoy nadando en la piscina". Entonces la persona a la derecha del facilitador tiene que simular lo que el facilitador dijo ósea que estaba nadando en una piscina, mientras dice que está haciendo algo muy diferente, de esta manera debe continuar la actividad hasta que todos participen.

Actividad 2. La silueta humana: esta actividad consiste en que los estudiantes formen cuatro grupos, el facilitador les pide que cada grupo construya una silueta humana en el suelo con todos los materiales que tengan a su alcance en ese momento, ya sean lapiceros, mochilas, cuadernos, etc. Ellos deben construir esta silueta en el suelo en 8 minutos deberán a serla lo más real y parecida a una persona, una vez terminado el tiempo los participantes deben dejar las siluetas hasta donde la hicieron y luego el facilitador con otra persona harán de jurados para calificar al ganador .esta dinámica permite observar la creatividad, la atención, la participación y el trabajo en equipo.

\section{Actividad de Desarrollo}

Se realizan preguntas acerca del contenido del taller. Los participantes deben planificar una simulación de un problema que surge y como se resuelve con el trabajo de equipo

Reflexionar acerca de las ventajas del trabajo en equipo. Cuáles conductas deben estar presentes en el trabajo en equipo. Resumir los resultados en un organizador de información, puede ser un dibujo que lo realice cada grupo.

Actividad de cierre: Se realiza la evaluación de la dinámica se resumen los logros de la sesión de trabajo se presentan las conclusiones o acuerdos. 


\section{Sesión 6. Medios tecnológicos}

Objetivo: Reflexionar acerca del papel de la tecnología en el proceso de aprendizaje. Fortalecer el uso adecuado del teléfono celular, la computadora y la conexión a internet.

Contenido: Tecnologías de la Información y la Comunicación (TIC), Tecnología de Aprendizaje y la Comunicación (TAC), Tecnología del Empoderamiento y la participación (TEP). Motores de búsqueda, repositorios. Videos educativos. Construcción de productos educativos a través de la tecnología: blogs, wiki, podcast, Instagram, twitter.

Tiempo: $60^{\prime}$

Participantes: 25 estudiantes de quinto de secundaria.

Materiales y recursos: Marcadores, papel, computadora, presentaciones, teléfonos celulares, lista de repositorios y motores de búsqueda.

\section{Actividad de Inicio}

El facilitador inicia con una dinámica para generar ambiente cooperativo y de atención.

\section{Actividad de Desarrollo}

Se realizan preguntas acerca del contenido del taller. Realizar una búsqueda, evaluación y selección de una información, procesarla y difundirla a través de una plataforma tecnológica.

Planificar una simulación, son periodistas o médicos y necesitan una información específica para realizar una noticia o resolver un problema.

Reflexionar acerca de las ventajas de los repositorios y motores de búsqueda. Criterios para seleccionar algún material de formato electrónico. Importancia de la generación de contenidos. Responsabilidad del generador de contenidos en internet.

Actividad de cierre: Se realiza la evaluación de la dinámica se resumen los logros de la sesión de trabajo se presentan las conclusiones o acuerdos.

\section{CONCLUSIONES}

La atención en clase de los estudiantes del quinto de secundaria es muy baja; la dimensión participación oral se caracteriza porque los estudiantes no hacen preguntas acerca de la temática que se desarrolla y tienen dificultades para realizar resúmenes y emitir opiniones. Responden preguntas si el docente toma en cuenta la respuesta para una evaluación.

La dimensión de actividades escritas queda definida por el bajo interés que tienen los estudiantes para tomar apuntes, esta situación la resuelven usando el celular para dejar registros de las clases. En general cumplen con la realización de las actividades escritas dentro del aula.

Los estudiantes no tienen control de los distractores del entorno, mantienen conversaciones mientras el docente desarrolla la temática, usan el celular en clases.

Algunos de los factores que inciden en la baja atención en clase son: carencias en la comunicación entre docentes y estudiantes, estrategias de enseñanza pasivas, falta de normas para la convivencia en el aula y falta de motivación e interés por parte de los estudiantes durante las clases.

La propuesta de estrategia didáctica atiende las necesidades de los docentes y a los estudiantes con actividades que generan aprendizaje significativo y promueven la comprensión de la atención en clase. Se fundamenta en principios de la psicología, la pedagogía y la didáctica. 


\section{REFERENCIAS}

Barca, A. (2005). Atención a la diversidad de la educación secundaria en Galicia: Perfiles del alumnado con bajo rendimiento escolar y propuestas de intervención psicoeducativas. Revista galeno de ensino, 45, 353 - 385

Boujon, Ch., y Quaireau, Ch., (1999). Atención, aprendizajeyrendimiento escolar.Aportaciones de la Psicología cognitiva y experimental. Madrid: Narcea

Gascón, de la, A. y Paredes, J. (2008). Didáctica general: La práctica de la enseñanza en Educación Infantil, Primaria y Secundaria. España: McGraw Hill

Gazzaniga, M, Ivry, M.R, y Mangun, G.R. (2013). Cognitive Neuroscience: The Biology of the Mind. New York, W.W. Norton

Hernández, G. (2012). Procesos Psicológicos Básicos. Tlalnepantla, México: Red Tercer Milenio

LaBerge, D. (1995). Computational and anatomical models of selective attention in object identification. En Gazzaniga, Michael S. (Ed). The cognitive neurosciences. (pp. 649-663)
Lupón, M., Torrents, A. y Quevedo, L. (2012). Apuntes en psicología en atención visual. [Documento en línea] Barcelona, España: Universidad Politécnica de Catalunya. https://upcommons. upc.edu/handle/2117/189580

Navas Ara, M. (2010) Métodos diseños y técnicas de investigación pedagógica. Madrid: Universidad Nacional de Educación a Distancia

Osorio, A. (2012). Pedagogía y didáctica. Serie de innovación IDEP. Bogotá: Editorial Jotamar

Pérez, E. (2008). Desarrollo de los procesos atencionales. Madrid: E-Prints Complutense. https://eprints.ucm.es/id/eprint/8447/

Syka, J. y Merzenich, M. (2005). Plasticity and signal representation in the auditory system. New York: Springer

Vaello Orts, J. (2011) ¿Cómo dar clases a los que no quieren? Barcelona España: Grao

Van de Heijden, A. (1992). Selective attention in vision. New York: Routlege 